\title{
Remote-Controlled Release of Singlet Oxygen by the Plasmonic Heating of Endoperoxide-Modified Gold Nanorods: Towards a Paradigm Change in Photodynamic Therapy
}

\author{
Safacan Kolemen, Tugba Ozdemir, Dayoung Lee, Gyoung Mi Kim, Tugce Karatas, \\ Juyoung Yoon,* and Engin U. Akkaya*
}

\begin{abstract}
The photodynamic therapy of cancer is contingent upon the sustained generation of singlet oxygen in the tumor region. However, tumors of the most metastatic cancer types develop a region of severe hypoxia, which puts them beyond the reach of most therapeutic protocols. More troublesome, photodynamic action generates acute hypoxia as the process itself diminishes cellular oxygen reserves, which makes it a selflimiting method. Herein, we describe a new concept that could eventually lead to a change in the 100 year old paradigm of photodynamic therapy and potentially offer solutions to some of the lingering problems. When gold nanorods with tethered endoperoxides are irradiated at $808 \mathrm{~nm}$, the endoperoxides undergo thermal cycloreversion, resulting in the generation of singlet oxygen. We demonstrate that the amount of singlet oxygen produced in this way is sufficient for triggering apoptosis in cell cultures.
\end{abstract}

$T_{\text {he photodynamic therapy (PDT) of cancer has been }}$ considered a promising therapeutic approach for decades. ${ }^{[1]}$ Principal requirements for photodynamic action were established by the experiments of Raab and von Tappeiner at the turn of the 20th century. ${ }^{[2]}$ Based on their work, which was followed up by many others, ${ }^{[3,4]}$ a photosensitizer that can be excited by light in the visible, but more preferably in the red or near-IR region of the spectrum could sensitize groundstate molecular oxygen and generate a short-lived, cytotoxic reactive oxygen species, that is, singlet oxygen $\left(\mathrm{O}_{2}:{ }^{1} \Delta_{\mathrm{g}}\right)$. This process is inherently regioselective, as the singlet-oxygen generation will take place only in the region at which the light beam is directed. In combination with the enhanced permeation and retention (EPR) effect, ${ }^{[5]}$ which leads to sensitizer

[*] Dr. S. Kolemen, Dr. T. Ozdemir, Prof. E. U. Akkaya

UNAM-Institute of Material Science and Nanotechnology

Bilkent University

Ankara 06800 (Turkey)

E-mail: eua@fen.bilkent.edu.tr

D. Lee, G. M. Kim, Prof. J. Yoon

Department of Chemistry and Nano Science

Ewha Womans University

Seoul 120-750 (Korea)

E-mail: jyoon@ewha.ac.kr

T. Karatas, Prof. E. U. Akkaya

Department of Chemistry, Bilkent University Ankara 06800 (Turkey)

(2) Supporting information and the ORCID identification number(s) for the author(s) of this article can be found under http://dx.doi.org/10. 1002/anie.201510064. accumulation in tumors, this non-invasive, or minimally invasive, treatment protocol, with tolerable side effects and a bonus of enhanced immune response, ${ }^{[6]}$ has tremendous therapeutic potential. However, the full promise of PDT has not been realized except perhaps for some niche applications such as superficial lesions. ${ }^{[7]}$ The limited applicability is not necessarily due to the lack of optimal sensitizers or smart delivery/activation processes $;{ }^{[8-15]}$ the problem unfortunately lies at the core of the PDT paradigm. First, even at the optimum wavelengths, the tissue penetration of light is very ineffective beyond the first few millimeters. ${ }^{[16]}$ The second issue is oxygen concentration. Most tumors develop a hypoxic region, and this is more common in aggressive metastatic tumors. ${ }^{[17]}$ Such hypoxic tissues are highly resistant to chemotherapy and radiotherapy. ${ }^{[18]}$ PDT, on the other hand, is inhibited even more severely, because normoxic oxygen concentrations are essential for effective singlet-oxygen generation. Furthermore, even in normal tissues, the PDT process itself decreases the cellular oxygen concentration,
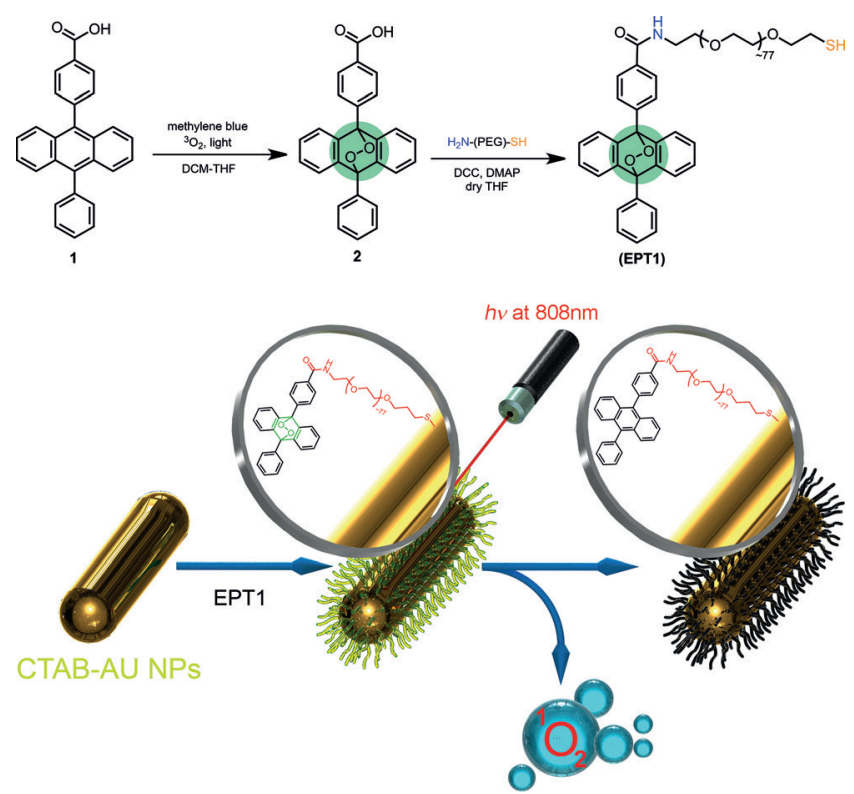

Figure 1. New photodynamics concept. Top: Synthesis of the targeted anthracene endoperoxide derivative (EPT1) for gold nanorod functionalization. The PEG linker enhances the water solubility of both the nanorods and the anthracene derivative. Bottom: Plasmonic heating of the gold nanorods at near-IR wavelengths leads to thermal cycloreversion of the tethered endoperoxides yielding singlet oxygen. 
thus inhibiting the therapeutic process. ${ }^{[19]}$ Clearly, in most applications, PDT becomes a self-limiting methodology.

Herein, we propose that if singlet oxygen could be generated directly in the desired region by remotely controlled mechanisms, all of these issues would be successfully circumvented.

Endoperoxides are the most reliable chemical source of singlet oxygen. Endoperoxides of naphthalene, anthracene, and a few other arenes were shown to generate singlet oxygen upon warming, with good chemical yields. ${ }^{[20]}$ The kinetic parameters for endoperoxide cycloreversion versus oxidative decomposition have been studied, ${ }^{[21]}$ and there are reported examples of relatively stable endoperoxides that cleanly undergo cycloreversion upon heating, generating singlet oxygen. ${ }^{[22-24]}$

Whereas there are other methods for remote-controlled heating ${ }^{[2,26]}$ for our proof of principle, we chose to make use of the plasmonic heating of metallic nanoparticles, specifically that of gold nanorods. Based on literature precedence, ${ }^{[27]}$ and targeting an aspect ratio of four, it is possible to prepare gold nanorods with strong near-IR absorption (longitudinal peak). Then, appropriate functionalization with endoperoxides should yield nanorods that can release singlet oxygen upon irradiation with near-IR light as a result of plasmonic heating. Singlet-oxygen generation in organic or aqueous solvents through the decomposition of endoperoxides within a biological context has been reported previously. ${ }^{[28-30]}$ However, our work is the first ever to demonstrate a viable process that could replace PDT in cell cultures and uses tissue-penetrating near-IR irradiation.

Kinetic stability at ambient temperature (or $37^{\circ} \mathrm{C}$ ) is important. The half-lives of anthracene 9,10-endoperoxides are typically years at room temperature, ${ }^{[31]}$ but when heated, they decompose rapidly, mostly by cycloreversion. With these considerations, we targeted the synthesis of a thiol-terminated and water-soluble 9,10-disubstituted anthracene, EPT1 (Figure 1; for details of its synthesis, see the Supporting Information). Endoperoxide $\mathbf{2}$ can be prepared by the reaction of compound $\mathbf{1}$ with oxygen gas bubbled through the DCM/THF solution in the presence of methylene blue as a photosensitizer under irradiation with a broadband white lamp (200 W). Then, compound 2 was subjected to a DCC coupling with amine-PEG-thiol to yield EPT1.
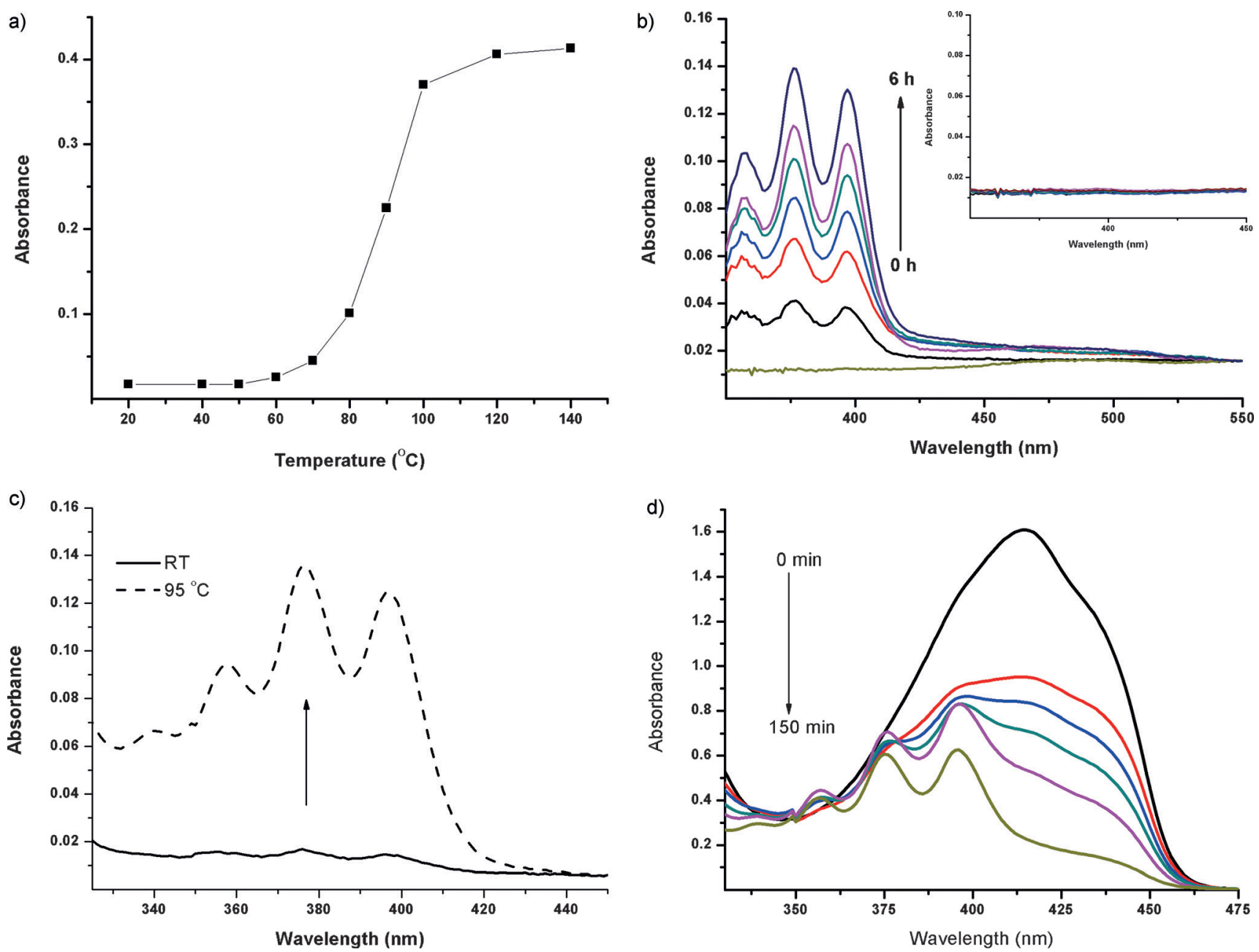

Figure 2. Thermal cycloreversion of EPT1. a) Absorbance at one of the anthracene peaks ( $404 \mathrm{~nm}$ ) after heating EPT1 for 30 min at the indicated temperatures. b) Thermolysis of EPT1 in DMSO at $95^{\circ} \mathrm{C}$ for $6 \mathrm{~h}$. Inset: Absorbance of EPT1 in DMSO at $37^{\circ} \mathrm{C}$. Samples were heated for $6 \mathrm{~h}$. c) When EPT1 is heated in HEPES buffer ( $\mathrm{pH} 7.2,20 \mathrm{~mm}$ ), the typical anthracene spectral signature is observed. d) Decrease in absorbance of DPBF in DMSO in the presence of EPTI upon heating at $70^{\circ} \mathrm{C}$ and reappearance of the parent anthracene absorption bands. 
Our first investigation was a chemical assessment of the decomposition-cycloreversion process. We demonstrated that upon heating in DMSO or HEPES buffer, the endoperoxide cleanly regenerates the parent anthracene, as confirmed by the typical electronic absorption spectrum of anthracene (Figure 2). Next, it was equally important to demonstrate that singlet oxygen is among the products of the cycloreversion. By using the selective singlet-oxygen trap 1,3-diphenylisobenzofuran (DPBF), we were able to detect singlet-oxygen formation (Figure 2d) during the thermal decomposition of the endoperoxide in DMSO, as the trap absorbance decreased with a concomitant increase in the intensities of the characteristic anthracene peaks.

For the other component of the therapeutic agent, gold nanorods with the desired characteristics $(40 \mathrm{~nm}$, aspect ratio: 4) were prepared following literature precedence. ${ }^{[27]}$ TEM images of the CTAB (cetyltrimethylammonium bromide) stabilized nanorods confirmed the characteristics of the prepared material (Figure 3). Then, the nanorods were treated with the thiol-functionalized epoxide EPT1 in HEPES buffer ( $\mathrm{pH} 7.2,20 \mathrm{~mm}$ ), followed by centrifugation to separate the functionalized nanorods. After the removal of any unreacted epoxide EPT1, the thermal decomposition of nanorod-tethered EPT1 (EPT1-GNR) was studied. Quantitative analysis of the spectroscopic data reveals that each GNR carries about $6.5 \times 10^{9}$ EPT1 molecules (see the Supporting Information).

As the temperature of the DMSO solution containing the singlet-oxygen trap DPFB and the EPT1-GNR conjugate was increased, the DPBF peak around $414 \mathrm{~nm}$ decreased in intensity. When the same solution was irradiated with a laser at $830 \mathrm{~nm}$ at room temperature, a decrease in the absorbance of the singlet-oxygen trap was observed, whereas for GNRs alone, no changes were observed when irradiated in the same manner (Figure 4).

Finally, we wanted to demonstrate the viability of the GNR-endoperoxide agent as a remote-controlled source of singlet oxygen in cell cultures. To that end, HeLa cells were incubated with EPT1-GNR or the control compound GNRPEG (GNRs with a PEG-SH ligand) for $24 \mathrm{~h}$. Cryo-TEM imaging shows that the EPT1-GNRs are located inside the cells within vacuoles (see the Supporting Information, Figure S5). As an additional control, a singlet-oxygen-quencher azide $(10 \mathrm{~mm})$ was added to the cell media in one series. To detect singlet-oxygen generation, cells were stained with Cyto-ID ${ }^{\circledR}$ oxidative-stress detection reagents for $30 \mathrm{~min}$ after irradiation with an $808 \mathrm{~nm}$ NIR laser $\left(2.0 \mathrm{~W} \mathrm{~cm}^{-2}\right)$ for $10 \mathrm{~min}$, and then the cell images were acquired.
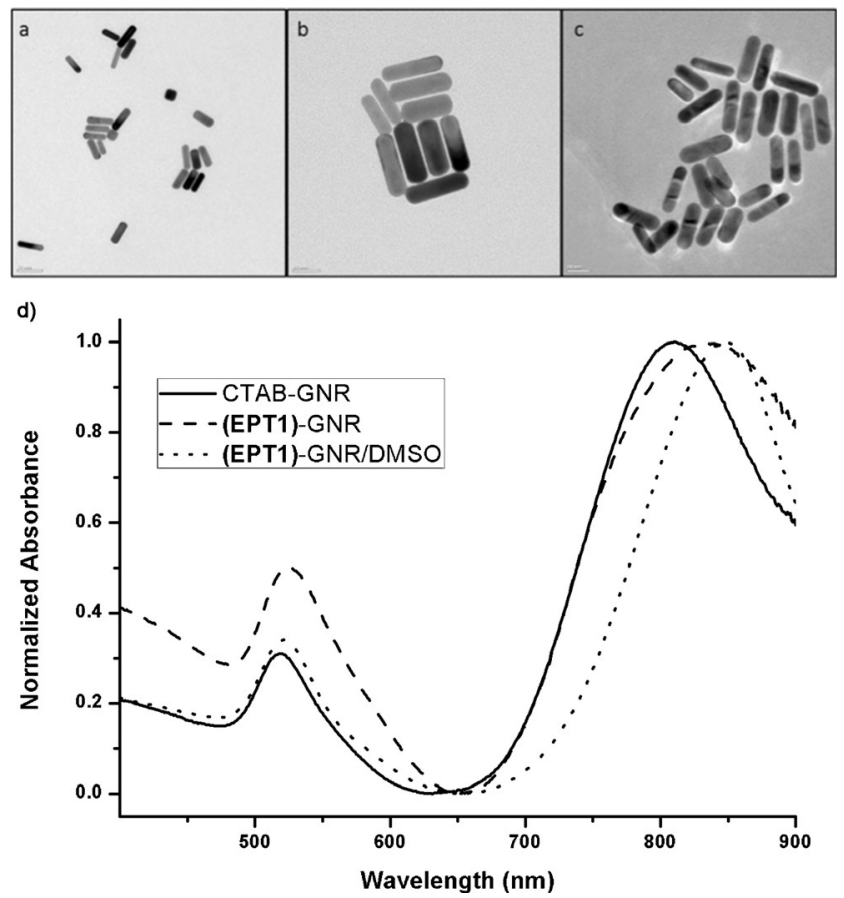

Figure 3. TEM images of the CTAB-GNR conjugates $(a, b)$ and the EPT1-GNR conjugate (c). d) Normalized electronic absorption spectra of CTAB-GNR and EPT1-GNR in HEPES ( $\mathrm{pH} \mathrm{7.2,20} \mathrm{mm)} \mathrm{and} \mathrm{of}$ EPT1-GNR in DMSO.
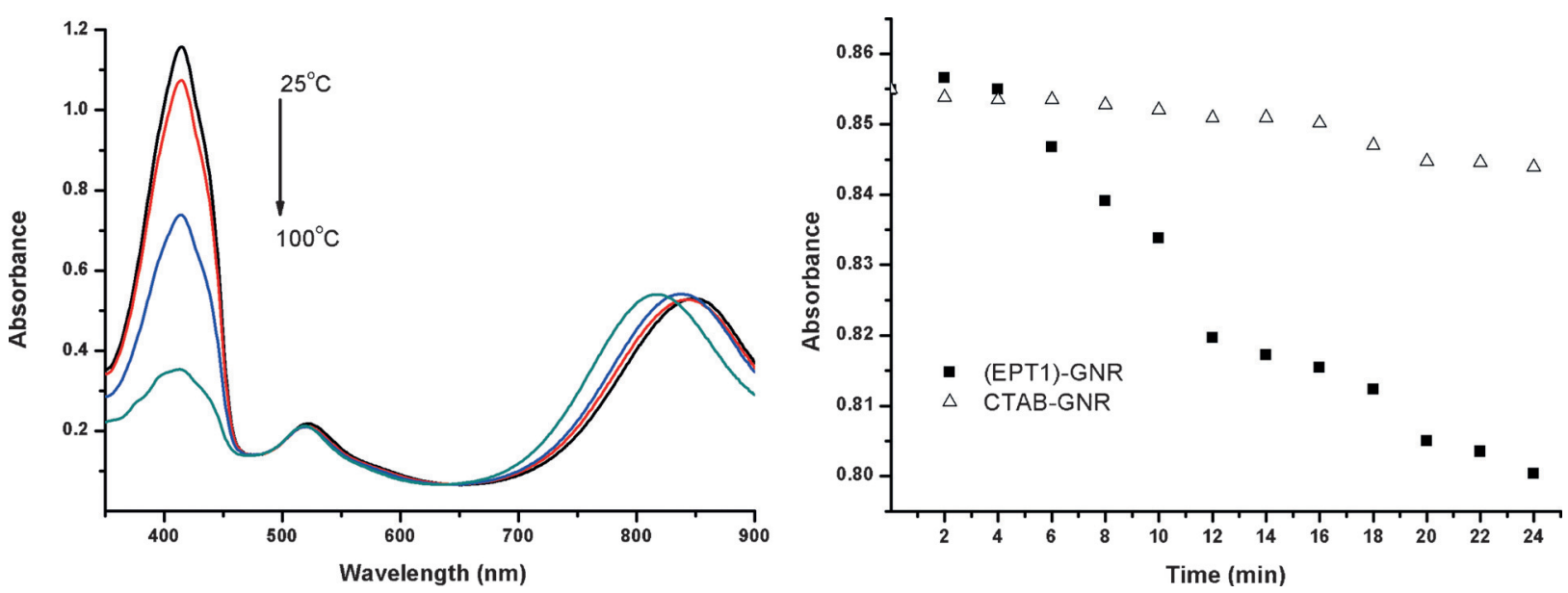

Figure 4. Decomposition and singlet-oxygen generation of plasmonically heated EPT1-GNR conjugates. Left: Decrease in the absorbance at $414 \mathrm{~nm}$ of DPBF in DMSO in the presence of EPT1-GNR upon heating in an oil bath. Right: Relative ${ }^{1} \mathrm{O}_{2}$ generation efficiency of EPT1-GNR and CTAB-GNR only in DMSO, detected by the absorbance decrease of DPBF at $414 \mathrm{~nm}$ with time. Excitation was at $830 \mathrm{~nm}\left(1.0 \mathrm{~W} \mathrm{~cm}^{-2}\right)$. During the first $4 \mathrm{~min}$, the samples were kept in the dark. 

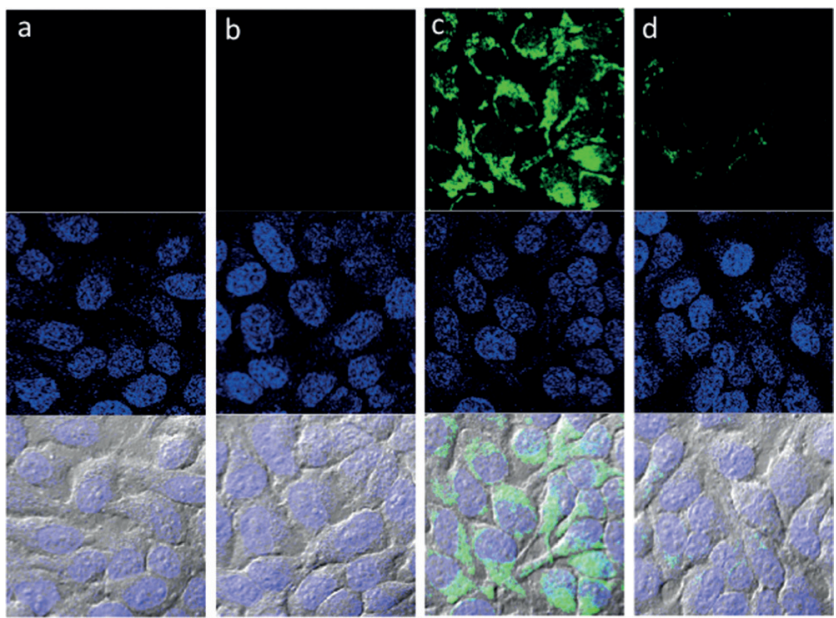

Figure 5. ROS generation by EPT1-GNR in cell cultures. HeLa cells were incubated with no additive (a), GNR-PEG (10 pM; b), EPT1GNR (10 pM; c), or EPT1-GNR (10 pM) and $\mathrm{NaN}_{3}(10 \mathrm{~mm}$; d). All cells were irradiated with a $808 \mathrm{~nm}$ laser $\left(2.0 \mathrm{Wcm}^{-2}, 10 \mathrm{~min}\right)$ and incubated with an ROS sensor (Cyto-ID ${ }^{\circledR}$ oxidative-stress detection kit) for $30 \mathrm{~min}$. The cell images were acquired by confocal microscopy. Top: ROS sensor; middle: DAPI; bottom: merged with DIC.

The confocal microscopy results are highly revealing and in accordance with our expectations (Figure 5). The control compound GNR-PEG did not generate oxidative stress in the cell cultures when irradiated at a fluence rate of $2.0 \mathrm{~W} \mathrm{~cm}^{-2}$ (Figure 5). EPT1-GNR irradiation, however, caused fluorescence emission in the cytosol, lighting up the reactive oxygen sensor. Inhibition by the added azide (Figure $5 \mathrm{~d}$ ) identified the reactive oxygen species as singlet oxygen.

In a similar experiment, cells undergoing apoptosis were identified with fluorescently labeled Annexin V (Figure 6). Corroborating the ROS results, only the irradiation of cell
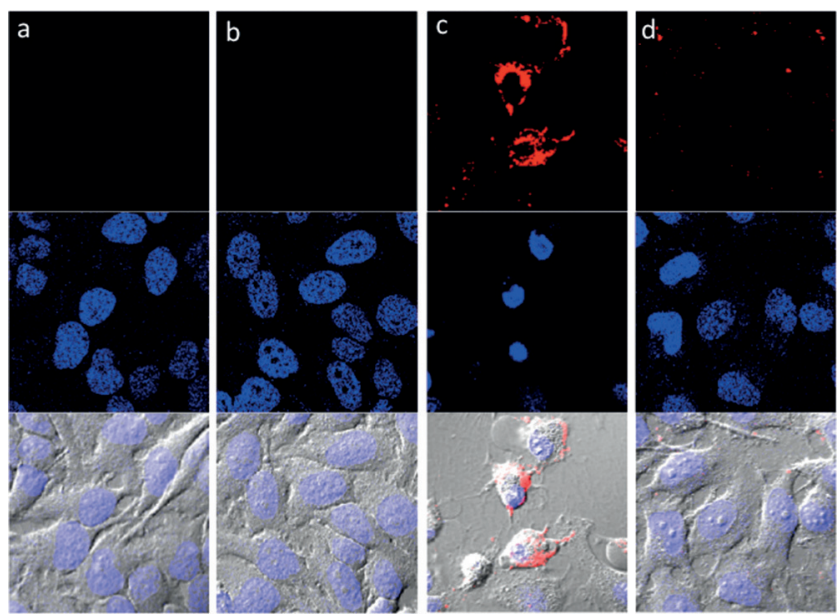

Figure 6. Apoptosis induced by EPT1-GNR in cell cultures. HeLa cells were incubated with no additives (a), GNR-PEG (10 pM; b), EPT1GNR (10 pM; c), or EPT1-GNR (10 pM) and $\mathrm{NaN}_{3}(10 \mathrm{~mm}$; d). All cells were irradiated with a $808 \mathrm{~nm}$ laser $\left(2.0 \mathrm{~W} \mathrm{~cm}^{-2}, 10 \mathrm{~min}\right)$, incubated for $16 \mathrm{~h}$, and stained with Annexin $\mathrm{V}$ and DAPI for $20 \mathrm{~min}$. The cell images were acquired by confocal microscopy. Top: Annexin $\mathrm{V}$; middle: DAPI; bottom: merged with DIC. cultures containing EPT1-GNR resulted in the fluorescence labeling of the outer leaf of the cell membranes, an indication of apoptosis. Again, the addition of azide resulted in a very large suppression of cell death. MTT [3-(4,5-dimethylthiazol2-yl)-2,5-diphenyltetrazolium bromide] colorimetric assays (Figure 7) of metabolic activity provided a quantitative picture supporting the same conclusion. A low concentration of near-IR-irradiated EPT1-GNR provides singlet oxygen, which could initiate apoptotic cell death in HeLa cell cultures. Cell viability increases with the added azide, again demonstrating the fact that cell death is due to the generation of singlet oxygen.

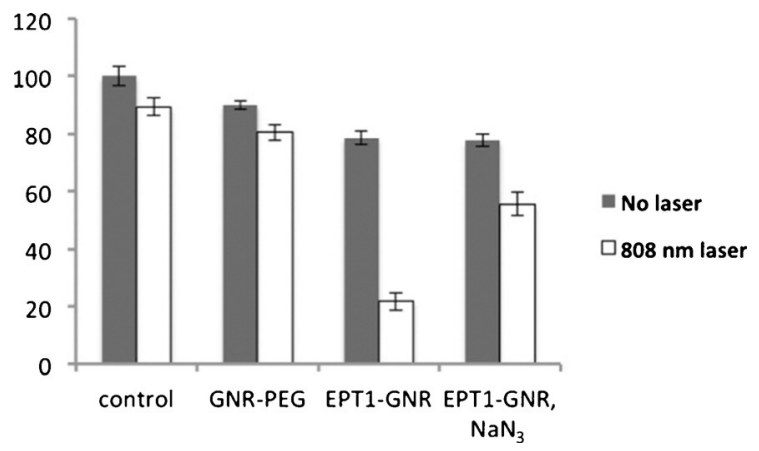

Figure 7. MTT assays of cell viability. HeLa cells were incubated with 10 pм of GNR-PEG or EPT1-GNR for $24 \mathrm{~h}$, washed with DPBS, and irradiated with a $808 \mathrm{~nm}$ laser $\left(2.0 \mathrm{Wcm}^{-2}, 10 \mathrm{~min}\right)$. After $24 \mathrm{~h}$, the cell viability was determined by an MTT test.

In conclusion, we have demonstrated that chemically generated singlet oxygen can induce apoptosis and lead to the death of cancer cells in cell cultures. The photodynamic therapy of cancer, which rests on the success of the photosensitized generation of singlet oxygen in tumors, has been stunted by the problems of tumor hypoxia (both induced and chronic) and light penetration, confining it to superficial lesions. The chemical generation of singlet oxygen by the controlled cycloreversion of endoperoxides has the potential to alleviate these very critical problems. It is clear that even a small quantity of singlet oxygen is capable of creating a significant apoptotic response, most likely through amplification mechanisms. Thus the fact that endoperoxides are stoichiometric agents does not present a problem. A near-IR light source and gold nanorods were used in this proof-ofprinciple study, but the thermal cycloreversion of endoperoxides can also be remotely triggered by alternating magnetic fields by using endoperoxides tethered to iron oxide nanoparticles.

We believe that more than one century after the first reports on photodynamic therapy, its principal tenet is on the verge of a radical change. The controlled chemical generation of singlet oxygen may improve the application and availability of endoperoxide-based therapies (EPT). Having its roots in PDT, EPT may deliver all of the promises of PDT without its inherent limitations. Work to realize these goals is in progress in our laboratories. 


\section{Acknowledgements}

E.U.A. gratefully acknowledges support from Bilkent University. J.Y. acknowledges a grant from the National Research Foundation of Korea (NRF) funded by the Korean government (MSIP, 2012R1A3A2048814). We are grateful to Bora Bilgic for the graphic design.

Keywords: endoperoxides - nanorods - photodynamic therapy · photosensitizers $\cdot$ singlet oxygen

How to cite: Angew. Chem. Int. Ed. 2016, 55, 3606-3610 Angew. Chem. 2016, 128, 3670-3674

[1] P. Agostinis, K. Berg, K. A. Cengel, T. H. Foster, A. W. Girotti, S. O. Gollnick, S. M. Hahn, M. R. Hamblin, A. Juzeniene, D. Kessel, M. Korbelik, J. Moan, P. Mroz, D. Nowis, J. Piette, B. C. Wilson, J. Golab, CA Cancer J. Clin. 2011, 61, 250-281.

[2] D. E. Dolmans, D. Fukumura, R. K. Jain, Nat. Rev. Cancer 2003 , $3,380-387$

[3] T. J. Dougherty, J. E. Kaufman, A. Goldfarb, K. R. Weishaupt, D. Boyle, A. Mittleman, Cancer Res. 1978, 38, 2628-2635.

[4] J. Moan, Q. Peng, Anticancer Res. 2003, 23, 591-600.

[5] H. Maeda, J. Wu, T. Sawa, Y. Matsumura, K. Hori, J. Controlled Release 2000, 65, 271-284.

[6] A. P. Castano, P. Mroz, M. R. Hamblin, Nat. Rev. Cancer 2006, 6 , $535-545$.

[7] C. A. Morton, K. E. McKenna, L. E. Rhodes, Br. J. Dermatol. 2008, 159, 1245-1266.

[8] M. Bio, P. Rajaputra, G. Nkepang, Y. You, J. Med. Chem. 2014, 57, $3401-3409$.

[9] A. Shao, Y. Xie, S. Zhu, Z. Guo, S. Zhu, J. Guo, P Shi, T. D. James, H. Tian, W. H. Zhu, Angew. Chem. Int. Ed. 2015, 54 , 7275-7280; Angew. Chem. 2015, 127, 7383-7388.

[10] I. Simsek-Turan, F. Pir-Cakmak, D. C. Yildirim, R. Cetin-Atalay, E. U. Akkaya, Chem. Eur. J. 2014, 20, 16088-16092.

[11] S. Kolemen, M. Isik, G. M. Kim, D. Kim, H. Geng, M. Buyuktemiz, T. Karatas, X.-F. Zhang, Y. Dede, J. Yoon, E. U. Akkaya, Angew. Chem. Int. Ed. 2015, 54, 5340-5344; Angew. Chem. 2015, 127, 5430-5434.

[12] B. Wang, H. Yuan, Z. Liu, C. Nie, L. Liu, F. Lv, Y. Wang, S. Wang, Adv. Mater. 2014, 26, 5986-5990.
[13] E. J. Kim, S. Bhuniya, H. Lee, H. M. Kim, C. Cheong, S. Maiti, K. S. Hong, J. S. Kim, J. Am. Chem. Soc. 2014, 136, 13888-13894.

[14] G. Kong, G. Anyarambhatla, W. P. Petros, R. D. Braun, O. M. Colvin, D. Needham, M. W. Dewhirst, Cancer Res. 2000, 60, $6950-6957$.

[15] N. Huebsch, C. J. Kearney, X. Zhao, J. Kim, C. A. Cezar, Z. Suo, D. J. Mooney, Proc. Natl. Acad. Sci. USA 2014, 111, 9762 - 9767.

[16] S. Stolik, J. A. Delgado, A. Pérez, L. Anasagasti, J. Photochem. Photobiol. B 2000, 57, 90-93.

[17] R. Sullivan, C. H. Graham, Cancer Metastasis Rev. 2007, 26, 319 331.

[18] J. A. Bertout, S. A. Patel, M. C. Simon, Nat. Rev. Cancer 2008, 8 , 967-975.

[19] T. M. Busch, S. M. Hahn, S. M. Evans, C. J. Koch, Cancer Res 2000, 60, 2636-2642.

[20] J.-M. Aubry, C. Pierlot, J. Rigaudy, R. Schmidt, Acc. Chem. Res. 2003, 36, 668-675.

[21] H. H. Wasserman, K. B. Wiberg, D. L. Larsen, J. Parr, J. Org. Chem. 2005, 70, $105-109$.

[22] C. Pierlot, S. Hajjam, C. Barthelemy, J.-M. Aubry, J. Photochem. Photobiol. B 1996, 36, 31-39.

[23] J. J. Baumes, J. J. Gassensmith, J. Giblin, J.-J. Lee, A. G. White, W. J. Culligan, W. M. Leevy, M. Kuno, B. D. Smith, Nat. Chem. 2010, 2, 1025-1030

[24] S. Benz, S. Nötzli, J. S. Siegel, D. Eberli, H. J. Jessen, J. Med. Chem. 2013, 56, 10171-10182.

[25] X. Liu, H. Chen, X. Chen, Y. Alfadhl, J. Yu, D. Wen, Appl. Phys. Rev. 2015, 2, 011103.

[26] N. W. S. Kam, M. O'Connel, J. A. Wisdom, H. Dai, Proc. Natl. Acad. Sci. USA 2005, 102, 11600-11605.

[27] B. Nikoobakht, M. A. El-Sayed, Chem. Mater. 2003, 15, $1957-$ 1962.

[28] K. Otsu, K. Sato, Y. Ikeda, H. Imai, Y. Nakagawa, Y. Ohba, J. Fujii, Biochem. J. 2005, 389, 197-206.

[29] A. M. Asadirad, Z. Erno, N. R. Branda, Chem. Commun. 2013, $49,5639-5641$.

[30] J.-J. Lee, A. Gonçalves, B. A. Smith, R. Palumbo, A. G. White, B. D. Smith, Aust. J. Chem. 2011, 64, 604-610.

[31] S. Martins, J. P. S. Farinha, C. Baleizao, M. N. Berberan-Santos, Chem. Commun. 2014, 50, 3317-3320.

Received: October 28, 2015

Revised: December 7, 2015

Published online: February 4, 2016 